\title{
EMIGRACIÓN Y REPRESENTACIÓN ESPECIAL: EVIDENCIA DE LOS ECUATORIANOS RESIDENTES EN EL EXTERIOR
}

\author{
Emigration and special representation: \\ evidence from Ecuadorian living abroad
}

Sebastián Umpierrez De Reguero*
Régis Dandoy
Tatiana Palma*

\begin{abstract}
Resumen. La mayoría de países del mundo han otorgado derechos de ciudadanía a sus emigrantes, en particular el voto externo y la doble ciudadanía. Sin embargo, existe un sistema electoral relativamente nuevo que los países están gradualmente legislando e implementando: la representación especial (la representación política de los emigrantes en las legislaturas nacionales del país de origen). En la actualidad, 14 Estados en África, Europa y América Latina extienden derechos de representación directa a su población residente en el exterior. La representación especial es un fenómeno altamente relacionado con las discusiones contemporáneas en el ámbito de los estudios sobre migración. Un número creciente de trabajos busca dar explicaciones sobre por qué y cómo los países de origen lo están adoptando. En este artículo, examinamos la representación especial en Ecuador mediante un diseño de investigación cuantitativa que considera las percepciones de los emigrantes. Nuestro objetivo es determinar si existen posibles correspondencias entre la percepción de la necesidad de reservar asientos para los ecuatorianos no residentes y el compromiso migrante. Los resultados subrayan relaciones significativas en encuestados que tienen doble ciudadanía, son miembros de asociaciones civiles e indican deseos de retornar con la necesidad de reservar asientos en la Asamblea Nacional por y para la comunidad emigrante.
\end{abstract}

Palabras clave: elecciones, transnacionalismo, representación especial, Ecuador, migrantes.

Abstract. A vast majority of countries worldwide have granted citizenship rights to their emigrants, in particular external voting and dual citizenship. However, there is a relatively new migrant political transnational practice

* Universidad Casa Grande. Guayaquil, Ecuador.

** Waseda University. Tokio, Japón. 
that home-countries are gradually legislating and implementing: special representation, i.e. emigrant political representation in home-country national legislatures. Nowadays, 14 states in Africa, Europe and Latin America extend direct representation rights to their population residing abroad. Special representation is an interesting phenomenon, highly connected with contemporary discussions in the field of migration studies. Up to date a growing number of studies seek to provide explanations on why and how home-countries are adopting it. In this article, we examine the case of the Ecuadorian special representation through a quantitative research design that takes into account the perceptions of the emigrant voters. Our goal is to determine possible correspondences of this transnational policy via the perception of necessity of having seats reserved for Ecuadorians living abroad on the migrant engagement. Findings highlight significant relations between Ecuadorians who have dual citizenship, members of a civil association, and that indicate a willingness to return with the necessity of reserving seats in the National Assembly for the migrant community.

Keywords: elections, transnationalism, special representation, Ecuador, migrants.

\section{Introducción}

La globalización ha permitido que las prácticas políticas transnacionales en migración cobren cada vez más relevancia en los debates contemporáneos. En respuesta, los Estados a nivel internacional han ido otorgando de forma progresiva derechos de consultación, participación y representación a sus emigrantes con el fin de mantener, crear y/o fortalecer vínculos con ellos ${ }^{1}$. En la actualidad, son 14 los países que además de extender la franquicia del voto a sus ciudadanos no residentes, les aseguran su representación directa en las legislaturas de origen -la representación especial ${ }^{2}-$; entre ellos, Ecuador desde 2007.

En América Latina, Ecuador no es el pionero en adoptar este tipo de voto extra-territorial ni de implementar otras prácticas políticas como la doble ciudadanía ${ }^{3}$. Sin embargo, gracias principalmente al impulso que le ha dado el gobierno de Rafael Correa (2007-2017), creando entre otras instituciones y procesos, la Secretaria Nacional del Migrante (SENAMI), ahora Vice-Ministerio de Movilidad Humana, se ha consolidado en el presente como un ejemplo emblemático para estudiar problemáticas de la migración internacional desde un enfoque transnacional ${ }^{4}$.

\footnotetext{
LAFLEUR, Jean-Michel. Transnational politics and the State: The External Voting Rights of Diasporas.

2 COLLYER, Michael. Inside out? Directly elected 'special representation' of emigrants in national legislatures and the role of popular sovereignty.

3 Colombia, en efecto, viene trabajando en estos temas desde 1991, año de su última modificación constitucional.

4 BOCCAGNI, Paolo. Reminiscences, Patriotism, Participation: Approaching External Voting in Ecuadorian Immigration to Italy.
} 
Aunque existen investigaciones acerca de las interacciones entre los ciudadanos no residentes con el país de origen ${ }^{5}$, inclusive del caso ecuatoriano ${ }^{6}$, todavía los efectos que este sistema electoral de representación directa produce en los residentes en el exterior, no han sido satisfactoriamente tratados por los académicos. Considerando los antecedentes, se percibe la necesidad de desarrollar análisis empíricos menos descriptivos y más causales. De ahí que en este artículo se busque determinar posibles correspondencias entre el compromiso político de los ecuatorianos no residentes y la representación especial mediante un análisis cuantitativo, de tipo descriptivo-relacional.

Con este estudio, de corte transversal, se espera contribuir a caracterizar la representación especial en el caso ecuatoriano, a encontrar causalidades para conocer cómo se piensa al objeto de estudio desde el (potencial) votante, y en particular, a trazar patrones de comportamiento de los electores en torno a esta política diaspórica. Para estos fines, se utiliza una encuesta como única herramienta de recolección de datos, a través de un muestro probabilístico a 385 ecuatorianos no residentes. Las respuestas son analizadas mediante la prueba de ji-cuadrado ya que las variables de interés a tratar para las posibles relaciones, son nominales.

En las dos primeras secciones de este artículo se examina la aproximación teórico-conceptual del transnacionalismo, la noción de ciudadanía, y las características esenciales de la representación especial. Luego se estructura la investigación, se detallan los datos y el método a seguir, así como el perfil de la muestra. Finalmente, en los últimos apartados, se asume la parte empírica del estudio, en donde las correspondencias alternativas permiten delinear conclusiones para continuar con el estudio de la representación especial.

\section{Transnacionalismo y representación especial}

El transnacionalismo no ha emergido en la agenda internacional por condición espontánea. Los procesos de interconexión, cada vez más rápidos e intensos, no solo han transformado los discursos y la manera en cómo se

\footnotetext{
5 BAUBÖCK, Rainer. Stakeholder Citizenship and Transnational Political. Participation: A Normative Evaluation of External. Voting; COLLYER, Michael, VATHI, Zana. Patterns of extraterritorial voting; ESCOBAR et alii. Assessing Candidates at Home and Abroad: A Comparative Analysis of Colombian Expatriates in the 2010 Presidential Elections; LAFLEUR, Jean-Michel, ØSTERGAARD-NIELSEN, Eva, CIORNEI, Irina. Making the absent present: political parties and emigration issues in country of origin parliaments.

6 BOCCAGNI, Paolo. Migrants social protection as an transnational process: public policies and emigrant iniciative in the case of Ecuador; RAMÍREZ, Jacques. Construyendo más democracia: Análisis al sufragio migrante en Ecuador; CORTÉS-MASIONAVE, Almudena, SAN MARTíN, Anna. Transnacionalismo político: Políticas migratorias de vinculación de los estados de origen y de las asociaciones migrantes en España. Los casos ecuatoriano y colombiano; UMPIERREZ DE REGUERO, Sebastián, ORRANTIA, Daniela, JARA-ALBA, Carol. Una radiografía a las políticas transnacionales en migración desde la perspectiva del país de origen.
} 
entiende el mundo, las esferas sociales, políticas y económicas, sino también han logrado ubicar a la humanidad en otro tipo de discusiones teóricas ${ }^{7}$. Siguiendo los lineamientos del enfoque transnacional, concepciones como el Estado-nación ligado al territorio pierden importancia ante una posibilidad más grande que no se estructura a partir del estudio de A ni de B de manera independiente, sino de modo simultáneo. Los espacios transnacionales vienen a romper con la idea clásica de la geografía política, de las fronteras, de los límites abstractos, y en el caso de la migración internacional, adhieren tanto a la sociedad de acogida como de origen, al contrario de la asimilación ${ }^{8}$.

Aunque es inexistente el consenso con respecto al surgimiento del transnacionalismo, es plausible aseverar que el término en cuestión apareció entre las décadas de 1970 a 1990 con el cambio de paradigma del sistema internacional, en donde sobresale la emergencia de los actores no estatales ${ }^{9}$. Otras aproximaciones establecen una condición histórica y hallan evidencia en publicaciones y regulaciones jurídicas internacionales de fines del siglo XIX, principios del $X X^{10}$.

El transnacionalismo invita al reconocimiento de los migrantes como agentes sociales en el sistema internacional ${ }^{11}$ y no, como aquellos individuos en búsqueda de mejores oportunidades económicas, que han "traicionado" a sus patrias ${ }^{12}$. Bajo este escenario, el migrante puede ser percibido como una parte importante en la construcción de los Estados-nación contemporáneos, que puede llegar a influir en la definición de las instituciones políticas, económicas y sociales del país de origen ${ }^{13}$.

Así emerge el transnacionalismo político como resultado a un fuerte movimiento migratorio y al interés de definir las relaciones del Estado con los ciudadanos no residentes. Con este giro teórico, discusiones como las asociadas a la desterritorialización del Estado moderno, entran en vigor. De ahí que textos seminales ${ }^{14}$ expongan que los individuos hoy en día pueden residir en cualquier parte del mundo y seguir siendo parte activa del país que han dejado físicamente. De este modo se explica el surgimiento de conceptos como el

7 HELD, David. La democracia y el orden global; MURRAY, Warwick. Geographies of globalization.

8 LEVITT, Peggy, GLICK-SCHILLER, Nina. Conceptualizing Simultaneity: A Transnational Social Field Perspective on Society; GUARNIZO, Luis, PORTES, Alejandro, HALLER, William. Assimilation and Transnationalism: Determinants of Transnational Political Action among Contemporary Migrants.

9 KEOHANE, Robert, NYE, Joseph. Transgovernmental Relations and International Organizations.

${ }^{10}$ LAFLEUR, op. cit.; ØSTERGAARD-NIELSEN, Eva. Sending countries policies.

11 BASCH, Linda, GLICK-SCHILLER, Nina, BLANC-SZANTON, Cristina. Nations Unbound: Transnational Projects, Postcolonial Predicaments, and Deterritorialized Nation-States.

12 BERG, Ulla, TAMAGNO, Carla. El Quinto Suyo from above and from Below: State Agency and Transnational Political Practices among Peruvian Migrants in the US and Europe.

${ }^{13}$ CORTES-MASIONAVE, SANMARTIN, op. cit.

${ }^{14}$ COLLYER, VATHI, op. cit.; LAFLEUR, op. cit. 
transnacionalismo de Estado y la visibilización por parte de los gobiernos sobre la importancia de las comunidades de expatriados.

Efectivamente, la globalización ha impulsado una nueva mirada hacia las teorías tradicionales de Estado, sobre todo aquellas que comparten de cerca su origen con la noción de la ciudadanía republicana. Estas teorías simpatizan con el significado y los significantes del Estado moderno. En respuesta, surge la necesidad de una nueva concepción, de una renombrada noción de ciudadanía; una que se adapte a la realidad de un mundo en constante movimiento.

El modelo de ciudadanía tradicional (basada en el territorio) no se ajusta a la realidad generada por la movilización humana, que ha desencadenado una crisis del término desde comienzos de $1990^{15}$. Bajo este lineamiento, aparecen nuevas nociones como por ejemplo: la ciudadanía expandida ${ }^{16}$, aquella que tiende a ajustarse más a las significaciones contemporáneas, al abarcar dentro del mismo concepto no sólo al inmigrante sino también al emigrante. En paralelo, se acuñan otros adjetivos a lado de ciudadanía: como ciudadanía transnacional ${ }^{17}$ y ciudadanía extra-territorial ${ }^{18}$. Estos últimos modelos de ciudadanía se vinculan a una comunidad política sin una residencia fija, al igual que la ciudadanía expandida. En este sentido, se habla de una relación entre el individuo y el país de origen, así como un evidente divorcio entre su inherente definición y el territorio ${ }^{19}$. Es por eso que diversos autore ${ }^{20}$ insisten en el rol de los migrantes como participantes de dos espacios sociales: el país de origen y la sociedad de acogida.

Subsecuentemente, aparece el desafío de administrar esa extensión de derechos a través de políticas de Estado. Aquí entra en regla el análisis de las prácticas políticas transnacionales, aquellas que han sido motivo de múltiples estudios de caso y análisis comparativos en las últimas décadas ${ }^{21}$. Surgen las políticas de vinculación como las reformas ministeriales o consulares, las políticas de inversión, las facilidades para la canalización de remesas; por otra parte, la extensión de los servicios del Estado y protección; asimismo, se puede hablar de las políticas creadas para fortalecer el sentido de membrecía ${ }^{22}$. De estas prácticas políticas, nace la representación especial como resultado de las demandas de un mundo globalizado y donde las barreras geográficas ya no

${ }^{15}$ COLLYER, Michael. A geography of extra-territorial citizenship: explanation of external voting.

${ }_{16}$ BAUBÖCK, Rainer. Towards a political theory on migrant transnationalism.

17 IDEM. Transnational citizenship: membership and rights in international migration.

${ }^{18}$ COLLYER, op. cit.

19 Ibidem.

${ }^{20}$ LEVITT, GLICK-SCHILLER, op. cit.; VERTOVEC, Steven. Transnacionalismo migrante y modos de transformación.

${ }^{21}$ ØSTERGAARD-NIELSEN, op. cit.

${ }^{22}$ BAUBÖCK, Towards a political..., op. cit.; LEVITT, Peggy, DE LA DEHESA, Rafael. Transnational migration and the redefinition of the state: variations and explanations; ØSTERGAARD-NIELSEN, op. cit. 
figuran como limitante para que los emigrantes tengan voz y voto en la toma de decisiones de sus países de origen.

El término "representación especial" hace referencia al tipo de representación donde el representante comparte características tales como: etnia, religión, género, entre otras, con el constituyente ${ }^{23}$; en el caso de la migración, similares historias de vida. Esta característica, indudablemente descriptiva, juega un papel influyente en la percepción del votante hacia el representante; en específico, en la confianza y memoria del constituyente ${ }^{24}$.

Concerniente a su relación con los sistemas políticos, la representación especial está ligada a la estabilidad del sistema electoral ${ }^{25}$. Aunque la literatura sobre este fenómeno es aún limitada por la relativa novedad que encarna, se reconocen autores que han aportado significativamente a la conceptualización del término y han contribuido con estudios empíricos ${ }^{26}$. La representación especial produce una extensión de derechos que se ve reflejada en la voluntad del país de origen por la idea deontológica de mantener el vínculo con sus emigrantes. Es así como se abre la posibilidad de que los emigrantes puedan elegir representantes en el poder legislativo del país de origen.

El sistema de representación especial comprende dos características esenciales según la literatura: (1) los emigrantes pueden elegir asiento(s) a través del voto directo y (2) su representación es de carácter exclusivo; es decir, sólo para los emigrantes ${ }^{27}$. Portugal fue el primer caso de estudio en 1976. Actualmente, 13 países $^{28}$ más Niger, que en el primer trimestre de 2016 puso en práctica esta medida, extienden el sistema de representación especial para sus emigrantes. Esta tendencia es una categoría en crecimiento $^{29}$.

\section{Hipótesis}

En atención al objetivo de determinar posibles correspondencias entre la representación especial y el compromiso migrante se formula este apartado que revisa brevemente el estado de la cuestión sobre las prácticas políticas transnacionales en migración para plantear las hipótesis que guían este trabajo. Se consideran variables como la doble ciudadanía, los años de residencia en la sociedad de acogida, la membrecía a asociaciones civiles y filiación política, así como la esperanza de retorno y reinserción en el país de origen del migrante;

\footnotetext{
${ }^{23}$ MANSBRIDGE, Jane. Rethinking representation; WILLIAMS, Melissa. Voice, trust and memory: marginalized groups and the failings of liberal representation; YOUNG, Iris. Democracy and inclusion.

${ }^{24}$ REYNOLDS, Andrew. Reserved seats in national legislatures: a research note.

${ }^{25}$ COLLYER, Inside out?..., op. cit.

${ }^{26}$ Ibidem; LAFLEUR, op. cit.; ØSTERGAARD-NIELSEN, CIORNEI, op. cit.

27 Ibidem.

${ }^{28}$ Argelia, Cabo Verde, Colombia, Croacia, Ecuador, Francia, Italia, Macedonia, Mozambique, Portugal, República Dominicana, Rumania, Túnez.

${ }^{29}$ ØSTERGAARD-NIELSEN, CIORNEI, op. cit.
} 
esto esperando que las condiciones mencionadas causen variación en la necesidad de reservar asientos para los ciudadanos que residen en el exterior.

En este sentido, es pertinente indicar que de la ciudadanía extra-territorial se desprenden varios elementos, los más polémicos: el voto extra-territorial y el sistema de representación especial ${ }^{30}$. La posibilidad de seguir como ciudadano en el exterior, crea una serie de responsabilidades tanto para el Estado como para el individuo en su búsqueda de mantener un vínculo que traspase bordes geográficos.

Las tasas de participación electoral reflejan el compromiso migrante, su interés por elegir representantes y ser representados en el poder legislativo del país de origen. En Ecuador, todos los ciudadanos tienen obligatoriamente que ejercer su derecho al voto, salvo las personas con discapacidad y analfabetas, los jóvenes de 16 a 18 años, los miembros de las Fuerzas Armadas y Policía Nacional en servicio activo, los mayores de 65 años, incluyendo a los ecuatorianos residentes en el exterior, que sufragan de manera facultativa ${ }^{31}$. Se entiende por ende que el voto para el ciudadano residente es tanto un derecho como una obligación; mientras que, para el ecuatoriano no residente sólo un derecho ${ }^{32}$.

Al ser facultativo, la tasa de participación puede reflejar un compromiso político sesgado por la voluntad del país de origen. No obstante, hay autores que coinciden con la idea de Michael Collyer: "como ciudadanos no residentes, los emigrantes mantienen una relación con su país de origen que puede ser más importante para ellos que su rol como residente no ciudadano" ${ }^{\prime 3}$.

El crecimiento de una población que vive una doble vida va en aumento. Es progresivo el aumento de individuos que hablan dos idiomas, tienen casa en dos países (origen y acogida) y mantienen un vínculo transfronterizo ${ }^{34}$. Los migrantes pueden coexistir bajo un doble marco de referencia.

Al ser un efecto del transnacionalismo, la doble ciudadanía se la puede percibir como una ampliación de derechos o como un límite de los mismos. La ciudadanía de la sociedad de acogida le otorga al migrante el reconocimiento de nuevos derechos ${ }^{35}$, y a su vez, depende de la voluntad del país de origen para mantener sus derechos previos. En el presente, un migrante puede utilizar su ciudadanía de origen como detonador de reconocimiento y respeto por parte de las autoridades de la sociedad de acogida ${ }^{36}$. Estos derechos que derivan de

${ }^{30}$ COLLYER, VATHI, op. cit.

31 Art. 11 del CÓDIGO DE LA DEMOCRACIA.

${ }^{32}$ CONSTITUCIÓN DE LA REPÚBLICA DEL ECUADOR.

${ }^{33}$ COLLYER, A geography..., op. cit., p. 56.

${ }^{34}$ GUARNIZO, PORTES, HALLER, op. cit.

${ }^{35}$ SPIRO, Peter. Dual citizenship as human right.

${ }^{36}$ Como sucede con los británicos en España, según establece CIORNEI, Irina. Political parties and transnational communities. A contextual analysis of British and Romanian residents in Spain. 
las políticas transnacionales sea desde el país de origen o residencia, pueden ser varias: las facilidades y ayudas, inclusive económicas, que los gobiernos de origen les están otorgando a sus emigrantes para su retorno; la protección y asistencia por parte de los consulados en las distintas sociedades de acogida o las políticas de inversión y ahorro con acción afirmativa ${ }^{37}$. Este doble marco de referencia, permite asumir la siguiente hipótesis:

H1. La percepción de la necesidad de tener asientos reservados para los ciudadanos que residen en el exterior es menor entre los encuestados que tienen doble ciudadanía.

No obstante, los años que un migrante reside en la sociedad de acogida representan otra posible condición causal en el comportamiento transnacional. La asimilación e integración que un inmigrante tiene después de una serie de años en la sociedad de acogida es diferente de aquellos que recién emigraron. Tanto la literatura clásica ${ }^{38}$, como específica del caso ecuatoriano ${ }^{39}$, revela que mientras más asentado se encuentra el migrante en el país de acogida, más interesado está en la actividad transnacional.

Ahora bien, los años de residencia van acompañados de derechos y obligaciones tanto de la sociedad de acogida como del país de origen. De ahí que se pueda reflexionar acerca de al menos tres formas de comportamiento al permanecer por un periodo estable en la sociedad de acogida: una posibilidad podría conllevar su paulatino olvido de la política del país de origen; otra que la retome con más ahínco, dado sus sentimientos de patriotismo, nostalgia y deseos de retornar ${ }^{40}$; o la tercera, que considere los incentivos selectivos (derechos de la sociedad de acogida) que el migrante recibe para tomar la decisión de acercarse o no al país de origen ${ }^{41}$. En base al primer argumento de los arriba mencionados, se podría asumir lo siguiente:

H2. La percepción de la necesidad de tener asientos reservados para los ciudadanos no residentes es menor entre los encuestados que han permanecido un largo período de su vida adulta en el extranjero.

Por su parte, la creación de mecanismos que faciliten la participación política (electoral y no-electoral) de los emigrantes en los diferentes países de destino es un objeto de estudio que interesa a varios académicos ${ }^{42}$. Puntualmente,

${ }^{37}$ LEVITT, DE LA DEHESA, op. cit.; ØSTERGAARD-NIELSEN, op. cit.

38 PORTES, Alejandro. Convergencias teóricas y evidencias empíricas en el estudio del transnacionalismo de los inmigrantes.

39 BOCCAGNI, Reminiscences..., op. cit.

40 Ibidem.

${ }^{41}$ LACOMBA, Cristina. The role of language and the presence of previous immigration cohorts in immigrant political engagement: Ecuadorian collective action in New York City and Madrid.

${ }^{42}$ BAUBÖCK, op. cit.; LAFLEUR, op. cit.; ØSTERGAARD-NIELSEN, op. cit. 
se pueden subdividir estas prácticas en dos categorías: (1) las oficiales y (2) las no oficiales ${ }^{43}$. Las prácticas oficiales son coordinadas por las instituciones del Estado; mientras que las no oficiales se nutren de la participación de los actores no estatales, que se manifiestan generalmente en el surgimiento y desarrollo de movimientos sociales ${ }^{44}$. En efecto, actividades de negociación y cabildeo (lobbying) podrían ser un ejemplo emblemático de estas últimas prácticas.

Las actividades de negociación y cabildeo han sido históricas. En Estados Unidos se puede hacer referencia a las prácticas de influencia de las diásporas cubana e israelí en relación a la construcción de una política exterior con intereses claros para ambos grupos ${ }^{45}$. En Ecuador, quizás el caso más reciente de cómo una membrecía con el país de origen entra en juego se percibió en los esfuerzos de solidaridad de los ecuatorianos residentes en el exterior después del terremoto de fuerte magnitud telúrica del 16 de abril de $2016^{46}$.

Las actividades transnacionales informales de los emigrantes no son una reacción al aumento de las prácticas oficiales gubernamentales ${ }^{47}$. Estas actividades aparecen por iniciativa de la propia comunidad. Al demostrarse la relevancia social y económica de esta población, los gobiernos le empezaron a dar importancia ${ }^{48}$. Por estos motivos, se concuerda que las prácticas no oficiales influyen en el compromiso de los emigrantes con su país de origen ${ }^{49}$. En este sentido, se presupone la siguiente hipótesis:

H3. La percepción de la necesidad de tener asientos reservados para los ciudadanos que residen en el exterior es mayor entre los encuestados miembros de una asociación civil conformada por migrantes en el país de acogida.

Ahora bien, los esfuerzos por entender el funcionamiento de los partidos políticos en la realidad transnacional han sido recientes y aún reducidos ${ }^{50}$. Se conoce que los partidos políticos están cada vez más interesados en el rol del voto emigrante. Han creado sucursales en el exterior ${ }^{51}$ para tener una relación más directa con los electores no residentes. No obstante, existen en la actualidad

\footnotetext{
${ }^{43}$ COLLYER, A geography..., op. cit.

44 Ibidem.

45 LAFLEUR, op. cit.

${ }^{46}$ UMPIERREZ DE REGUERO, Sebastián, GONZÁLEZ-PAREDES, Santiago, VANONI, Stefano. Terremoto 7.8: respuestas de los Ecuatorianos residentes en el exterior.

47 PORTES, Alejandro, ESCOBAR, Cristina, RADFORD, Alexandria. Immigrant Transnational Organizations and Development: A Comparative Study.

${ }^{48}$ CORTÉS-MASIONAVE, SAN MARTIN, op. cit.

${ }^{49}$ FAIST, Thomas. Diaspora and transnationalism: what kind of dance partners?

${ }^{50}$ JAULIN, Thibaut. Geographies of external voting: the Tunisian elections abroad since the 2011 Upspring; CIORNEI, Irina, ØSTERGAARD-NIELSEN, Eva. Emigration and turnout. Determinants of non-resident citizen electoral mobilization in home country legislative elections.

${ }^{51}$ CIORNEI, ØSTERGAARD-NIELSEN, op. cit.
} 
Estados como Canadá que han prohibido la ejecución de campañas políticas y el asentamiento de oficinas de partidos políticos de otro país en su territorio ${ }^{52}$.

En Ecuador, este tema es doblemente interesante. La reglamentación de las organizaciones políticas ecuatorianas tiene una condición sui generis ${ }^{53}$ : los migrantes al disputar su participación electoral en circunscripciones especiales de representación directa, pueden crear movimientos políticos que los representen ${ }^{54}$. De esta manera, la membrecía o afiliación a un partido o movimiento político puede sumarse al compromiso político del migrante con su país de origen. De ahí que:

H4. La percepción de la necesidad de tener asientos reservados para los ciudadanos que residen en el exterior es mayor entre los encuestados afiliados a una organización política ecuatoriana.

Finalmente, el mantener la ciudadanía del país de origen puede generar que el emigrante llegue a sentir que no ha dejado su país de origen y por esta razón no expresaría necesariamente un deseo de regreso ${ }^{55}$. No obstante, y a pesar de que la migración ecuatoriana se caracteriza por ser de carácter permanente y sin retorno, logrando en un gran porcentaje obtener residencia legal en los países de destino ${ }^{56}$, hay referencias ${ }^{57}$ que hacen repensar el rol de los inmigrantes en actividades transnacionales y su deseo de retorno. Desde la creación de la SENAMI en 2009, se ha puesto al retorno en la agenda política ecuatoriana como un tema prioritario ${ }^{58}$. En esta línea, se puede formular la siguiente hipótesis:

H5. La percepción de la necesidad de tener asientos reservados para los ciudadanos que residen en el exterior tiene un impacto positivo entre los encuestados que desean retornar y reinsertarse en el país de origen.

\section{Datos y métodos}

Esta investigación estudia el caso de la representación especial ecuatoriana desde el potencial votante mediante un enfoque metodológico cuantitativo. El objetivo es determinar si existen posibles correspondencias entre la percepción de la necesidad de reservar asientos para los ecuatorianos no residentes y el compromiso migrante. En este sentido, las variables a considerar son la

\footnotetext{
52 LAFLEUR, op. cit.

${ }^{53}$ Art. 2 y 4 del CODIFICACIÓN DEL REGLAMENTO PARA LA INSCRIPCIÓN DE PARTIDOS, MOVIMIENTOS POLÍTICOS Y REGISTRO DE DIRECTIVAS.

${ }^{54}$ UMPIERREZ DE REGUERO, ORRANTIA, JARA-ALBA, op. cit.

${ }^{55}$ BRUNEAU, Michel. Diaspora, transnational spaces and communities.

${ }^{56}$ CORTES-MASIONAVE, SANMARTÍN, op. cit.

57 BOCCAGNI, op. cit.; RAMÍREZ, op. cit.

${ }^{58}$ Ver el PLAN RETORNO creado por la SENAMI en 2009.
} 
percepción de la necesidad de tener asientos reservados para los ciudadanos que residen en el exterior (variable dependiente) y el compromiso migrante (variable independiente) por parte de los emigrantes, medido desde la filiación a organizaciones políticas ecuatorianas, membrecía a asociaciones civiles conformadas por migrantes, años de residencia en la sociedad de acogida y doble ciudadanía.

Toda esta información es extraída utilizando una encuesta online como única herramienta de recolección de datos. Los cuestionarios fueron posteados en páginas webs pertenecientes a asociaciones civiles de ecuatorianos residentes en el exterior. Se incluyó también el instrumento en la plataforma digital Internations, a la cual tienen acceso miles de ecuatorianos.

El instrumento ha estado posteándose desde mediados de octubre de 2016, fecha en la que se inició el registro de candidaturas presidenciales y legislativas para el proceso electoral, hasta el 2 de abril de 2017 (segunda vuelta presidencial). En particular, se seleccionó el caso de la representación especial en Ecuador justamente por el contexto de estabilidad/cambio que se presenta en estas últimas elecciones.

Se debe indicar como ventajas de aplicar una encuesta online en este periodo: (1) el interés político que puede ser mayor en elecciones que sin ellas; (2) se ofrece mayor flexibilidad de respuesta; y (3) se ahorra en costos de movilización. Por el contrario, como debilidad se podría señalar la dificultad para recolectar los datos dada la población geográficamente dispersa ${ }^{59}$. En cualquier caso, la unidad de análisis es el universo de ciudadanos no residentes de Ecuador -los 1.144.408 ecuatorianos ${ }^{60}$ distribuidos en 55 países de acogida ${ }^{61}-$.

La muestra es de diseño probabilístico finito estratificado proporcional, tomando a las 55 sociedades de acogida como un solo universo y ponderando las respuestas por los segmentos poblacionales que corresponden a las tres circunscripciones especiales del exterior que Ecuador ha creado para organizar las elecciones extra-territoriales desde $2009^{62}$. El cálculo de población y muestra

\footnotetext{
${ }_{59}$ UMPIERREZ DE REGUERO, Sebastián, DÁVILA, Pamela. Interviewing by Internations: a methodological discussion to explore special representation.

${ }^{60}$ La ex SENAMI indicaba a comienzos de 2013 que el número de ecuatorianos y sus descendientes fuera del país estaba calculado en una cifra que oscilaba entre 2 y 3 millones de personas (ANDES, 12 de enero de 2013). Hoy en día, el Vice-Ministerio de Movilidad Humana habla de un rango de entre 1 y 2 millones de ecuatorianos no residentes.

${ }^{61} \mathrm{NACIONES}$ UNIDAS. Tendencias de la población migrante internacional: migrantes por destino y origen.

${ }^{62}$ El artículo 150(3) de la LEY ORGÁNICA ELECTORAL Y DE ORGANIZACIONES POLÍTICAS: CÓDIGO DE LA DEMOCRACIA establece la creación de tres circunscripciones especiales del exterior para organizar los procesos electorales en el exterior: (1) Canadá y Estados Unidos; (2) Europa, Asia y Oceanía; y (3) Latinoamérica, El Caribe y África.
} 
contará con un nivel de confianza del 95\%, un error muestral del 5\% y una tasa de heterogeneidad del $50 \%$. A partir de esto se establece que la muestra de población encuestada es de 385 personas, y el coeficiente de estratificación es de 0,00033642 (ver Cuadro 1).

\section{CUADRO 1 - Muestra de ecuatorianos residentes en el exterior por circunscripción}

\begin{tabular}{ccccc}
\hline Circunscripción & Población & $\%$ & Muestra & $\%$ \\
\hline América Latina, El Caribe y África & 83.322 & 7,28 & 28 & 7,27 \\
Canadá y Estados Unidos & 488.674 & 42,70 & 164 & 42,60 \\
Europa, Asia y Oceanía & 572.412 & 50,02 & 193 & 50,13 \\
Totales & 1.144 .408 & 100,00 & 385 & 100,00 \\
\hline
\end{tabular}

Fuente: elaboración propia en base a datos de Naciones Unidas (2013).

Por su parte, el cuestionario está estructurado en preguntas cerradas en torno a cuatro secciones: (1) el perfil socio-demográfico del encuestado; (2) la experiencia migratoria; (3) la experiencia política en el país de origen; y (4) la experiencia política en la sociedad de acogida. Las opciones de respuesta están diseñadas en torno a variables nominales y ordinales. De hecho, la variable dependiente, la necesidad de reservar asientos en el poder legislativo del país de origen, es de medición nominal (ver Figura 1).

\section{FIGURA 1 - ¿Cree usted que los ecuatorianos residentes en el exterior deben tener representantes en la Asamblea Nacional de Ecuador?}



Fuente: elaboración propia.

A pesar de que casi tres cuartas partes de la muestra han señalado la necesidad de reservar asientos en el poder legislativo ecuatoriano, puede presentarse como paradójicas sus percepciones sobre las posibles causalidades asociadas a la representación especial. La media de respuestas indica "indiferencia" $<<3,00>>$ en los 10 enunciados que se presentan a continuación (ver Cuadro 2). Si bien el promedio de repuestas parece no tener 
una postura clara, los coeficientes de desviación estándar puntualizan que las respuestas usuales oscilan entre un "en desacuerdo" $<<2,00>>$ y un "de acuerdo" $<<4,00>>$, lo que tendría más sentido.

CUADRO 2 - Percepciones de la representación especial

\begin{tabular}{lcc}
\hline & Media & $\begin{array}{c}\text { Desviación } \\
\text { estándar }\end{array}$ \\
\hline Impulsa el sentimiento de pertenencia & 2,91 & 1,403 \\
Mantiene conectados a los no-residentes con lo que pasa en Ecuador & 3,04 & 1,437 \\
Las autoridades de Ecuador solo hacen esto para obtener más remesas & 2,76 & 1,305 \\
Mejora la calidad de vida de los no-residentes en el país de acogida & 2,55 & 1,322 \\
Incluye a los no-residentes en las decisiones de Ecuador & 2,87 & 1,420 \\
De esta forma agradece por ayudar a la economía del país & 2,63 & 1,267 \\
Es simplemente para obtener votos & 2,83 & 1,325 \\
Motiva a regresar a vivir a Ecuador & 2,58 & 1,279 \\
Es eficiente para mejorar las leyes y políticas migratorias ecuatorianas & 2,88 & 1,402 \\
Es un gasto de dinero & 2,44 & 1,274 \\
\hline
\end{tabular}

Fuente: elaboración propia.

El perfil socio-demográfico de la muestra tiene predominancia femenina, sigue los parámetros de estratificación poblacional arriba-mencionados, y se concentra en los rangos etarios de 18-25 y de 26-33 (ver Cuadro 3).

CUADRO 3 - Perfil socio-demográfico de la muestra

\begin{tabular}{|c|c|c|c|c|c|c|}
\hline \multirow{2}{*}{$\begin{array}{l}\text { Género: } \\
\text { Circunscripción: }\end{array}$} & \multicolumn{2}{|c|}{$\begin{array}{l}\text { Femenino } \\
(57,3 \%)\end{array}$} & \multicolumn{4}{|c|}{$\begin{array}{l}\text { Masculino } \\
(42,7 \%)\end{array}$} \\
\hline & \multicolumn{2}{|c|}{$\begin{array}{l}\text { América Latina, El } \\
\text { Caribe y África } \\
(7,3 \%)\end{array}$} & \multicolumn{2}{|c|}{$\begin{array}{l}\text { Canadá y Estados } \\
\text { Unidos } \\
(42,6 \%)\end{array}$} & \multicolumn{2}{|c|}{$\begin{array}{l}\text { Europa, Asia y Oceanía } \\
(50,1 \%)\end{array}$} \\
\hline Rango de edad: & $\begin{array}{l}17-25 \\
(38,7 \%)\end{array}$ & $\begin{array}{l}26-33 \\
(20,0 \%)\end{array}$ & $\begin{array}{l}34-41 \\
(14,8 \%)\end{array}$ & $\begin{array}{l}42-49 \\
(12,2 \%)\end{array}$ & $\begin{array}{l}50-57 \\
(8,6 \%)\end{array}$ & $\begin{array}{l}58-82 \\
(5,7 \%)\end{array}$ \\
\hline
\end{tabular}

Fuente: elaboración propia.

En cuanto a la frecuencia de participación electoral extra-territorial, son pocos los ciudadanos no residentes que siempre ejercen su voto desde el exterior. Por el contario, la respuesta mayoritaria parece indicar la no participación electoral (Figura 2). 


\section{FIGURA 2 - Experiencia de voto en el exterior de la muestra}



Fuente: elaboración propia.

Concerniente a las preocupaciones que tal vez pueden tener los emigrantes ecuatorianos sobre su país de origen, se reconoce entre las opciones más frecuentes a la situación económica y las elecciones de 2017. La esperanza de retorno y reinserción en Ecuador parece ser una preocupación secundaria.

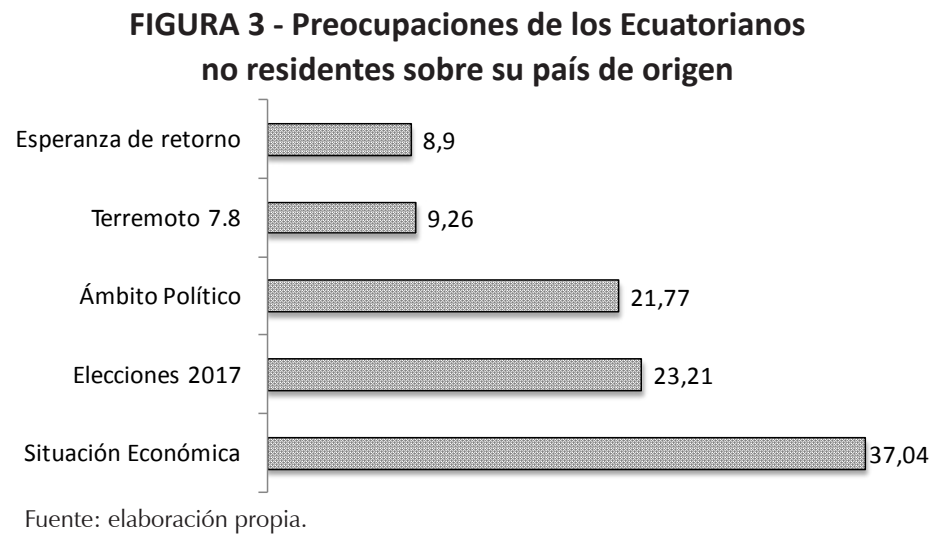

El método cuantitativo para el análisis y presentación de resultados es el ji-cuadrado. Se asume esta decisión metodológica al ser las variables de interés: nominales. Con este método se espera comprobar las cinco hipótesis arriba mencionadas.

\section{Resultados}

La estructura de este apartado va de la mano con el planteamiento de hipótesis y el diseño de investigación expuesto. Se trabaja con las variables $X$ que conforman la dimensión compromiso migrante (doble ciudadanía, membresía a asociaciones civiles, filiación política, años de residencia en la sociedad de 
acogida y esperanza de retorno) y se espera encontrar una correspondencia alterna o nula con la variable $Y$ acerca de la necesidad de reservar asientos en la Asamblea Nacional para los emigrantes, mediante la prueba del ji-cuadrado. Para ello, tanto la $Y$ como las $X$ son variables que están operacionalizadas como dummy. Esto se debe, la mayoría de veces, al tipo de respuesta que se buscaba con el cuestionario (No $<<0>>$ y Sí $<<1>>$ ), salvo en el caso de los años de residencia que a pesar de haber sido una variable escala en un principio, se la transforma en dummy también (de 1 a 10 años $<<1>>$, de 11 años ad infinitum $<<0>>$ ) para propósitos de este estudio. Así se comprueban/falsean las cinco hipótesis fijadas con anterioridad.

Entonces, ¿existe una relación entre la doble ciudadanía de los ecuatorianos residentes en el exterior y la necesidad (percibida) de representación especial? Con esta interrogante se pretende establecer si hay correspondencia entre que los ecuatorianos no residentes perciban mayores beneficios e incentivos en la sociedad de acogida a través del reconocimiento de ciudadanía, y si están de acuerdo con la necesidad de adoptar una provisión específica para representar a los emigrantes en las legislaturas de origen. De la muestra, quienes declaran tener doble ciudadanía representan el 50,91\%.

\section{CUADRO 4 - Frecuencias observadas y esperadas entre doble ciudadanía y representación especial}

\begin{tabular}{llll}
\hline \multirow{2}{*}{ Representación especial $(Y)$} & \multicolumn{2}{l}{ Doble Ciudadanía $(X)$} \\
\cline { 2 - 4 } & No & Sí & Totales por renglón \\
\hline No & $48(55,5)$ & $65(57,5)$ & $113(113)$ \\
Sí & $141(133,5)$ & $131(138,5)$ & $272(272)$ \\
Totales por columnas & $189(189)$ & $196(196)$ & $385(385)$ \\
\hline
\end{tabular}

$\mathrm{X}^{2}=2,799$

Fuente: elaboración propia.

Al tomar en cuenta la tabla de contingencia (ver Cuadro 4), se puede verificar que existen diferencias relevantes entre las frecuencias esperadas $y$ observadas, por lo que sería correcto asumir la hipótesis alternativa $\left(\mathrm{H}_{\mathrm{A}}=X>\right.$ Y). La prueba de ji-cuadrado así como las pruebas de validación (Phi y $\vee$ de Cramer) corroboran esta decisión. En efecto, el $X^{2}=2,799$ supera el ji-crítico de 2,71, así como el $\alpha=0,1$ es superior al valor $p=0,094$; esto, tomando un grado de libertad (por la matriz de 2x2) y un nivel de confianza del 95\%.

Si bien la doble ciudadanía puede ser una de las prácticas políticas transnacionales más recurrentes, el rol de las asociaciones civiles es de suma importancia para el involucramiento político. De hecho, el caso de Esteban Melo, asambleísta alterno del periodo 2009-2013, reelecto en las elecciones 
de 2017 por la circunscripción especial de Europa, Asia y Oceanía, puede ejemplificar la importancia de las asociaciones civiles como plataforma política. En tal sentido, habrá relación entre la membrecía a una asociación civil y la necesidad de reservar asientos en el poder legislativo. De la muestra, quienes declaran pertenecer a una asociación civil, ya sea sindical, política, recreativa como socio-cultural, representan un 10,91\%.

Solo con observar la tabla de contingencia (ver Cuadro 5), se puede hablar de una posible correspondencia entre las dos variables de interés; las frecuencias observadas no se asemejan con los recuentos esperados.

\section{CUADRO 5 - Frecuencias observadas y esperadas entre membrecía a asociaciones civiles y representación especial}

\begin{tabular}{llll}
\hline \multirow{2}{*}{$\begin{array}{l}\text { Representación } \\
\text { especial }(\mathrm{Y})\end{array}$} & \multicolumn{3}{l}{ Membrecía asociación civil $(\mathrm{X})$} \\
\cline { 2 - 4 } & No & Sí & Totales por renglón \\
\hline No & $107(100,7)$ & $6(12,3)$ & $113(113)$ \\
Sí & $236(242,3)$ & $36(29,7)$ & $272(272)$ \\
Totales por columnas & $343(343)$ & $42(42)$ & $385(385)$ \\
\hline$X^{2}=5,160$ & &
\end{tabular}

$X^{2}=5,160$

Fuente: elaboración propia.

Puntualmente, el $X^{2}=5,160$ supera el ji-crítico de 3,84, así como el $\alpha=$ 0,05 es superior al valor $p=0,023$; esto, tomando un grado de libertad (por la matriz de $2 \times 2$ ) y un nivel de confianza del $95 \%$. Bajo este escenario, se rechaza la $\mathrm{H}_{0}$ y se acepta la $\mathrm{H}_{\mathrm{A}}$ de relación entre la membrecía de asociación civil y la necesidad (percibida) de la representación especial. Se supone entonces que los ecuatorianos no residentes que se encuentran registrados en una asociación civil, están más dispuestos a realizar prácticas políticas transnacionales que les otorguen un mayor vínculo con el país de origen como la provisión de reservar asientos en la Asamblea Nacional para la comunidad migrante.

Las asociaciones civiles pueden subdividirse por temáticas o intereses muy diversos, desde deportes hasta actividades políticas; sin embargo, tienen un objetivo en común que es unir o crear espacios para compartir entre ecuatorianos. Estos espacios pueden promover actividades de participación colectiva, posiblemente ofrezcan información sobre la logística de procesos electorales, ayuden a otros connacionales en torno a los servicios sociales que pueden recibir en la sociedad de acogida y también en Ecuador, entre otras facilidades. En momentos de emergencia, pueden activarse y convertirse en contingentes de solidaridad o grandes aliadas en la recuperación de su país de 
origen, como se puede corroborar en el caso del terremoto de 2010 en Haití, tejiendo redes transnacionales ${ }^{63}$.

Al igual que la membrecía a las asociaciones civiles, para el compromiso migrante es importante la posibilidad de mantener, e inclusive adherirse una vez afuera, a un partido o movimiento político del país de origen. Se parte de la misma intuición que se acabó de comprobar en los párrafos anteriores con la membrecía a las asociaciones civiles: el que un ecuatoriano residente en el exterior decida afiliarse o mantener su filiación política de Ecuador, puede indicar compromiso. Ésta es una realidad que una gran cantidad de países progresivamente están aceptando y que su estudio está tomando una importancia creciente, como ya se mencionó. Es pertinente entonces preguntar: ¿Existe una correspondencia entre la filiación política por parte de los ecuatorianos residentes en el exterior con la idea de tener representantes emigrantes en el Poder Legislativo unicameral ecuatoriano? De la muestra, quienes aseguran constar como afiliados a una organización política ecuatoriana representan un $4,68 \%$.

Al tomar en cuenta la tabla de contingencia (ver Cuadro 6), se puede corroborar que en contraste a la doble ciudadanía y a la membrecía de asociación civil no produce una distancia significativa entre los recuentos esperados y observados. Los coeficientes arrojados por la prueba de ji-cuadrado y la corrección de Yates reafirman este dictamen.

\section{CUADRO 6 - Frecuencias observadas y esperadas entre filiación política y representación especial}

\begin{tabular}{llll}
\hline \multirow{2}{*}{ Representación especial $(Y)$} & \multicolumn{2}{l}{ Filiación política $(X)$} \\
\cline { 2 - 4 } & No & Sí & Totales por renglón \\
\hline No & $110(107,7)$ & $3(5,3)$ & $113(113)$ \\
Sí & $257(259,3)$ & $15(12,7)$ & $272(272)$ \\
Totales por columnas & $367(367)$ & $18(18)$ & $385(385)$ \\
\hline$X^{2}=1,465$ & &
\end{tabular}

Fuente: elaboración propia.

Ahora bien, no solo es una relación insignificante decir que la filiación política incide en la necesidad de reservar asientos en este ejercicio estadístico, sino que en términos de rigor científico no se cumple con el 100\% de las casillas con frecuencia menor esperada de 5 . Estas razones ponen de manifiesto que la hipótesis nula $\left(\mathrm{H}_{0}=X=Y\right)$ sería la más acertada. Se rechaza, por ende, la hipótesis alterna.

${ }_{63}$ LUNDY, Garvey. Transnationalism in the aftermath of the Haiti earthquake: reinforcing ties and second generation identity. 
La esperanza de retorno al Ecuador, por su parte, es quizás más recurrente para los migrantes de lo que puede parecer ${ }^{64}$. En ese marco, vale preguntarse: ¿Cómo se puede vincular esto a la representación especial?

Se puede asumir que personas con experiencia e historias similares con la migración, como los Asambleístas del Exterior, pueden conocer de mejor manera las prerrogativas de sus constituyentes no residentes y trabajar en respuesta de sus intereses. La Ley de Movilidad Humana, aprobada en 2017, es probablemente el mejor ejemplo para verificar la representación sustantiva de estos legisladores. Esta ley, al menos normativamente, no solo vela por la mejoría de los ecuatorianos residentes en el exterior sino que regula la inmigración en Ecuador y el retorno.

En este ejercicio, vale puntualizar planes de retorno elaborados por la SENAMI desde 2009 como "Bienvenidos a Casa" y el "Plan de Retorno para maestros ecuatorianos residentes en el exterior". Ambos, así como otras actividades de vinculación, buscaban atraer a los ecuatorianos residentes en el exterior con beneficios e incentivos, tanto materiales como simbólicos ${ }^{65}$. El primer plan incluía el pago por movilización del menaje de casa, la excepción de impuestos y acciones afirmativas en el sector público ecuatoriano.

\section{CUADRO 7 - Frecuencias observadas y esperadas entre esperanza de retorno y representación especial}

\begin{tabular}{llll}
\hline \multirow{2}{*}{ Representación especial $(Y)$} & \multicolumn{3}{l}{ Esperanza de retorno migrante $(X)$} \\
\cline { 2 - 4 } & No & Sí & Totales por renglón \\
\hline No & $100(91,3)$ & $13(21,7)$ & $113(113)$ \\
Sí & $211(219,7)$ & $61(52,3)$ & $272(272)$ \\
Totales por columnas & $311(311)$ & $74(74)$ & $385(385)$ \\
\hline$X^{2}=6,134$ & &
\end{tabular}

Fuente: elaboración propia.

De la misma manera que con la membrecía a las asociaciones civiles, existe una correspondencia alternativa entre la posibilidad de retorno y reinserción en el país de origen con la necesidad percibida de representación especial. De la muestra, quienes afirman el deseo de retornar representan el 19,22\%. Tanto la tabla de contingencia (ver Cuadro 7) como las pruebas de ji-cuadrado proponen negar la hipótesis nula y aceptar la hipótesis alterna. Efectivamente, el $X^{2}=6,134$ supera al valor del ji-crítico $=3,84$, así como el $\alpha=0,5$ no es inferior al valor $p=0,013$, tomando un grado de libertad (por la matriz de $2 \times 2$ ) y un nivel de confianza del 95\%.

\footnotetext{
${ }_{64}$ BOCCAGNI, op. cit.

${ }^{65}$ PALAZUELOS MANSO, Antonio, VILLAREAL VILLAMAR, María del Carmen. Más allá de las remesas: transformaciones socio-económicas y proyectos de migración y desarrollo en Ecuador.
} 
Finalmente, cabe preguntarse, y esto en consonancia con la literatura consultada $^{66}$, si existe una correspondencia entre los años de residencia del migrante en la sociedad de acogida y la necesidad (percibida) de la representación especial. La muestra indica los siguientes rangos (ver Figura 4):

\section{FIGURA 4 - Años en rangos de residencia}

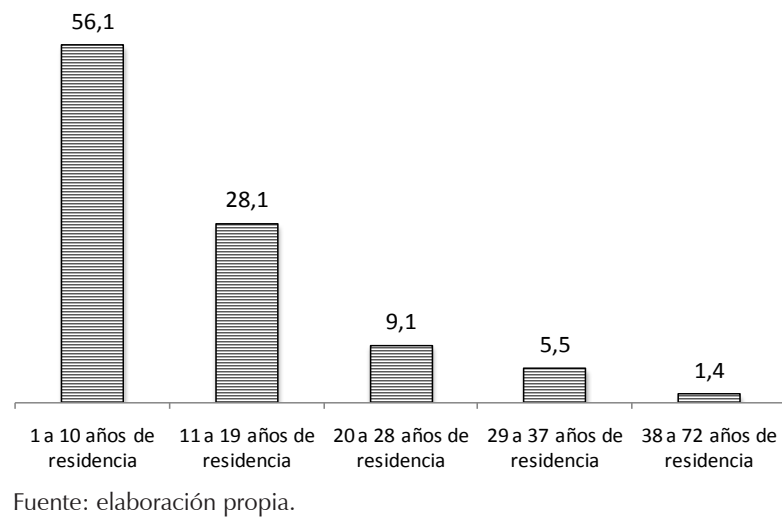

Al corroborar la tabla de contingencia (ver Cuadro 8), se identifica lo siguiente: (1) la diferencia artificial y que por propósitos de parsimonia en este artículo se ha tomado, entre los encuestados con menos años de residencia (de 1 a 10 años) y los que han residido por un tiempo más prologado en las diversas sociedades de acogida (de 11 ad infinitum); y (2) la posible relación entre la necesidad de reservar asientos y los años de residencia. Sin embargo, la pruebas de ji-cuadrado así como las pruebas de validación (Phi y V de Cramer) demuestran todo lo contrario. En consecuencia, se rechaza la hipótesis alterna y se acepta la nula (no hay correspondencia entre los años de residencia y la representación especial).

\section{CUADRO 8 - Frecuencias observadas y esperadas entre años de residencia en el exterior vs representación especial}

\begin{tabular}{llll}
\hline \multirow{2}{*}{ Representación especial $(\mathrm{Y})$} & \multicolumn{4}{l}{ Años de residencia $(\mathrm{X})$} \\
\cline { 2 - 4 } & $1-10$ & $11-70$ & Totales por renglón \\
\hline No & $54(49,6)$ & $59(63,4)$ & $113(113)$ \\
Sí & $115(119,4)$ & $157(152,6)$ & $272(272)$ \\
Totales por columnas & $169(169)$ & $216(216)$ & $385(385)$ \\
\hline
\end{tabular}

$X^{2}=0,984$

Fuente: elaboración propia.

${ }_{66}$ PORTES, Alejandro. Convergencias teóricas..., op. cit. 


\section{Conclusión}

El objetivo general de este artículo se estructura a partir de determinar si existen posibles correspondencias de la representación especial, vía la percepción de la necesidad de reservar asientos para los ciudadanos residentes en el exterior, en el compromiso migrante. El compromiso migrante parece ser multivariado. Se encuentran correspondencias alternativas entre los ecuatorianos no residentes encuestados que tienen doble ciudadanía (hipótesis 1), son miembros de asociaciones civiles (hipótesis 3) y esperan retornar para reinsertarse en Ecuador (hipótesis 5), con la necesidad de reservar asientos en la Asamblea Nacional por y para la comunidad migrante, mediante la prueba de ji-cuadrado. Todas estas tres hipótesis son verificadas a través de este análisis. La evidencia empírica contribuye al número creciente de contribuciones en materia de emigración y representación política, al comprobar/falsear hipótesis que se han utilizado previamente para abordar actividades transnacionales y políticas diaspóricas muy parecidas, como el voto externo. De este ejercicio empírico, también, se puede resaltar que los años de residencia (hipótesis 2) al contrario de lo que la literatura consultada advierte, al igual que la filiación política (hipótesis 4), en contraste a la intuición atada a resultados de ejercicios similares (el efecto de la membrecía a asociaciones civiles), son producto de relaciones nulas en el caso de estudio presentado. Ambas hipótesis son, por ende, rechazadas en este estudio.

La razón principal de elegir Ecuador es resultado de abordajes empíricos previos y sugerencias de la literatura. Puntualmente, Ecuador es señalado como un excelente caso de estudio para entender procesos migratorios y un ejemplo emblemático en la construcción de un Estado progresivamente más transnacional, en específico desde 2007 con la incumbencia de Alianza PAIS y la inclusión discursiva de la Quinta Región.

Por su parte, este artículo da cuenta de un diseño metodológico cuantitativo que innova en la recolección de los datos. Para estos fines, en este trabajo se utiliza una encuesta online posteada principalmente en páginas web de asociaciones civiles y en la plataforma de redes sociales Internations. Se obtuvo 385 respuestas, tomando como el universo a todos los países de acogida registrados en el censo de Naciones Unidas en 2013 que tuviesen ecuatorianos, y en paralelo, se estratificó esta muestra, de diseño probabilístico, por los segmentos poblacionales de las circunscripciones especiales que el Estado de Ecuador ha creado desde 2009. Indudablemente, esta decisión fue tomada en función de permitir una mayor flexibilidad de respuesta, ahorrar costos de movilización y porque la unidad de análisis es geográficamente dispersa. 
El compromiso migrante, efectivamente, se ve reflejado en distintas actividades como, por ejemplo la respuesta de la comunidad ecuatoriana frente a sucesos como el terremoto ocurrido el 16 de abril de 2016, o su presencia en grupos/comunidades de ecuatorianos en sitios de redes sociales como Facebook. Aunque no pueden ser percibidos como efectos directos de la representación especial justifican la existencia de la misma para albergar a una comunidad que demuestra su voluntad de participación con el país de origen. De hecho, los resultados de este documento indican relaciones de correspondencia entre la membrecía a las asociaciones civiles de migrantes como una clara evidencia de participación.

La representación especial se implementa con el objetivo de fortalecer el vínculo con el emigrante ecuatoriano. Este esfuerzo conlleva una responsabilidad para el Estado. La responsabilidad no sólo recae en los representantes por los ecuatorianos en el exterior en la Asamblea Nacional sino también en las instituciones del Estado involucradas en las prácticas políticas dirigidas en beneficio de la Quinta Región (Vice-Ministerio de Movilidad Humana). Una representación especial de calidad podría traducirse en la fortaleza de todos los niveles, tanto en la elaboración de leyes inclusivas que reflejen las necesidades y demandas de los emigrantes, así como la implementación de estrategias que favorezcan el proceso. Es importante, entonces, no solo la coordinación y comunicación entre los actores y la asignación de recursos que garantice la correcta implementación; sino también seguir investigando el tema en estudio.

Se recomienda hacer un análisis evolutivo, en donde se compare más de un proceso electoral como unidad de análisis. Asimismo, se sugiere la comparación con otros países que tengan el mismo sistema electoral para reservar asientos en legislaturas de origen, como Colombia o República Dominicana en la región latinoamericana, o Portugal, Túnez o Italia, casos influyentes al respecto. Finalmente y para futuras investigaciones, sería provechoso combinar métodos cuantitativos con cualitativos como estudios etnográficos para distinguir con más profundidad el objeto de estudio.

\section{Referencias}

ANDES. Cerca de 3 millones de ecuatorianos viven fuera del país. Disponible en: $<$ http://www.andes.info.ec/es/sociedad/cerca-3-millones-ecuatorianos-vivenfuera-pa\%C3\%ADs.html >.

ASAMBLEA NACIONAL. Constitución de la República del Ecuador. Quito-Ecuador, 2008.

BASCH, Linda; GLICK-SCHILLER, Nina; BLANC-SZANTON, Christina. Nations unbound: Transnational projects, postcolonial predicaments, and deterritorialized nation-states. London: Routledge, 1994. 
BAUBÖCK, Rainer. Transnational citizenship: membership and rights in international migration. Aldershot: Edward Elgar Publishing, 1994.

BAUBÖCK, Rainer. Towards a political theory of migrant transnationalism. International Migration Review, v. 37, n. 3, 2003, p. 700-723.

BAUBÖCK, Rainer. Stakeholder citizenship and transnational political participation: a normative evaluation of external voting. Fordham Law Review, v. 75, 2006, p. 2393-2447.

BERG, Ulla; TAMAGNO, Carla. El Quinto Suyo from above and from below: state agency and transnational political practices among Peruvian migrants in the US and Europe. Latino Studies, v. 4, n. 3, 2006, p. 258-281.

BOCCAGNI, Paolo. Migrants' social protection as a transnational process: public policies and emigrant initiative in the case of Ecuador. International Journal of Social Welfare, v. 20, n. 3, 2011, p. 318-325.

BOCCAGNI, Paolo. Reminiscences, patriotism, participation: approaching external voting in Ecuadorian immigration to Italy. International Migration, v. 49, n. 3, 2011, p. 76-98.

BOCCAGNI, Paolo; RAMÍREZ, Jacques. Building Democracy or Reproducing "Ecuadoreanness"? A Transnational Exploration of Ecuadorean Migrants' External Voting. Journal of Latin American Studies, v. 45, n. 4, 2013, p. 721-750.

BRUNEAU, Michel. Diasporas, transnational spaces and communities. In BAUBÖCK, Rainer; FAIST, Thomas (eds.), Diaspora and transnationalism: Concepts, theories and methods. Amsterdam: IMISCOE, 2010, p. 35-50.

CIORNEI, Irina. Political parties and transnational communities. A contextual analysis of British and Romanian residents in Spain. III Jornadas Políticas Migratorias, Justicia y Ciudadanía. Instituto de Filosofía, CSIC - Madrid, 27-29 octubre de 2010.

CIORNEI, Irina; ØSTERGAARD NIELSEN, Eva. Emigration and turnout. Determinants of non-resident citizen electoral mobilization in home country legislative elections. Congrès AFSP Aix, 2015.

COLLYER, Michael. A geography of extra-territorial citizenship: Explanations of external voting. Migration Studies, v. 2, n. 1, 2014, p. 55-72.

COLLYER, Michael. Inside out? Directly elected 'special representation' of emigrants in national legislatures and the role of popular sovereignty. Political Geography, v. 41, 2014, p. 64-73.

COLLYER, Michael; VATHI, Zana. Patterns of extra-territorial voting. Development Research Centre on Migration, Globalisation and Poverty Working Paper, v. 22, 2007, p. 1-36.

CONSEJO NACIONAL ELECTORAL. Ley Orgánica Electoral y de Organizaciones Políticas: Código de la Democracia. Quito-Ecuador, 2009.

CONSEJO NACIONAL ELECTORAL. Codificación del Reglamento para la Inscripción de Partidos, Movimientos Políticos y Registro de Directivas. Quito-Ecuador, 2013. 
CORTÉS-MAISONAVE, Almudena; SAN MARTÍN, Anna. Transnacionalismo político: políticas migratorias de vinculación de los estados de origen y de las asociaciones de migrantes en España. Los casos ecuatoriano y colombiano. XIV Encuentro de Latinoamericanistas Españoles, 2010, p. 1146-1164.

ESCOBAR, Cristina. Extraterritorial Political Rights and Dual Citizenship in Latin America. Latin American Research Review, v. 42, n. 3, 2007, p. 43-75.

ESCOBAR, Cristina; ARANA, Renelinda; MCCANN, James A. Assessing candidates at home and abroad: A comparative analysis of Colombian expatriates in the 2010 presidential elections. Latin American Politics and Society, v. 56, n. 2, 2014, p. 115-140.

FAIST, Thomas. Diaspora and transnationalism: What kind of dance partners? In BAUBÖCK, Rainer; FAIST, Thomas (eds.). Diaspora and transnationalism: Concepts, theories and methods. Amsterdam: IMISCOE, 2010, p. 9-34.

GUARNIZO, Luis Eduardo; PORTES, Alejandro; HALLER, William. Assimilation and Transnationalism: Determinants of Transnational Political Action among Contemporary Migrants. American Journal of Sociology, v. 108, n. 6, 2003, p. 1211-1248.

HELD, David. La democracia y el orden global. El estado moderno al Estado Cosmopolita. Barcelona: Paidos, 1997.

JAULIN, Thibaut et alii. The geography of external voting: the 2011 Tunisian election abroad. TSI Working Paper, 2015.

KEOHANE, Robert O.; NYE, Joseph S. Transgovernmental relations and international organizations. World Politics, v. 27, n. 1, 1974, p. 39-62.

LACOMBA, Cristina. The role of language and the presence of previous immigration cohorts in immigrant political engagement: Ecuadorian collective action in New York City and Madrid. Social Politics: International Studies in Gender, State \& Society, 2016, p. 1-19.

LAFLEUR, Jean-Michel. Transnational politics and the state: The external voting rights of Diasporas. London: Routledge, 2013.

LEVITT, Peggy; DE LA DEHESA, Rafael. Transnational migration and the redefinition of the state: Variations and explanations. Ethnic and Racial Studies, v. 26, n. 4, 2003, p. 587-611.

LEVITT, Peggy; SCHILLER, Nina Glick. Conceptualizing simultaneity: A transnational social field perspective on society. International Migration Review, v. 38, n. 3, 2004, p. 1002-1039.

LUNDY, Garvey. Transnationalism in the aftermath of the Haiti earthquake: Reinforcing ties and second-generation Identity. Journal of Black Studies, v. 42, n. 2, 2011, p. 203-224.

MANSBRIDGE, Jane. Rethinking representation. American Political Science Review, v. 97, n. 4, 2003, p. 515-528.

MURRAY, Warwick. Geographies of globalization. London: Routledge, 2005. 
NACIONES UNIDAS. Tendencias de la población migrante internacional: migrantes por destino y origen. Disponible en: < http://www.un.org/en/development/desa/ population/migration/data/estimates2/estimatesorigin.shtml>.

ØSTERGAARD-NIELSEN, Eva. Sending Country Policies. In GARCÉSMASCAREÑAS, Blanca; PENNINX, Rinus (eds.). Integration Processes and Policies in Europe. Springer International Publishing, 2016, p. 147-165.

ØSTERGAARD-NIELSEN, Eva; CIORNEI, Irina. Making the absent present: political parties and emigration issues in country of origin parliaments. Party Politics, 2017, p. 1-14.

PALAZUELOS MANSO, Antonio; VILLARREAL VILLAMAR, María del Carmen. Más allá de las remesas: Transformaciones socioeconómicas y proyectos de migración y desarrollo en Ecuador. Migración y Desarrollo, v. 11, n. 21, 2013, p.107-132.

PORTES, Alejandro. Convergencias teóricas y evidencias empíricas en el estudio del transnacionalismo de los inmigrantes. Migración y Desarrollo, n. 4, 2005, p. 2-19.

PORTES, Alejandro; ESCOBAR, Cristina; RADFORD, Alexandria Walton. Immigrant Transnational Organizations and Development: A Comparative Study. International Migration Review, v. 41, n. 1, 2007, p. 242-281.

RAMÍREZ, Jacques. Construyendo más democracia: análisis del sufragio migrante en Ecuador. Revista Democracias, v. 1, octubre-diciembre, 2013, p. 261-286.

REYNOLDS, Andrew. Reserved seats in national legislatures: A research note. Legislative Studies Quarterly, v. 30, n. 2, 2005, p. 301-310.

SENAMI. Plan Retorno. Quito-Ecuador, 2009.

SPIRO, Peter J. Dual citizenship as human right. International Journal of Constitutional Law, v. 8, n. 1, 2010, p. 111-130.

UMPIERREZ DE REGUERO, Sebastián; DÁVILA, Pamela. Interviewing by Internations: A methodological discussion to explore special representation in Mali. International Journal of Marketing, Communication and New Media, n. 1, 2016, p. 48-61.

UMPIERREZ DE REGUERO, Sebastián; ORRANTIA, Daniela; JARA-ALBA, Carol. Una radiografía de las políticas transnacionales en migración desde la perspectiva del país de origen. XII Conferencia INPAE: Estado, Políticas Públicas e Internacionalización, 2016.

UMPIERREZ DE REGUERO, Sebastián; GONZÁLEZ-PAREDES, Santiago; VANONI, Stefano. Terremoto 7.8: respuestas de los ecuatorianos residentes en el exterior. Congreso Internacional de Investigación en Ciencias Sociales, 2016.

VERTOVEC, Steven. Migrant transnationalism and modes of transformation. International Migration Review, v. 38, n. 3, 2004, p. 970-1001.

WILLIAMS, Melissa S. Voice, trust, and memory: Marginalized groups and the failings of liberal representation. Princeton: Princeton University Press, 2000.

YOUNG, Iris Marion. Democracy and inclusion. Oxford: Routledge, 2000. 


\section{Anexos:}

\section{CUADRO 9 - Lista de preguntas usadas (variables dependiente más independientes)}

\begin{tabular}{ll}
\hline 1. & ¿En qué año se fue de Ecuador? \\
\hline 2. & ¿Cuál es actualmente su país de acogida? \\
\hline 3. & ¿En qué año llegó a tu actual país de acogida? \\
\hline 4. & ¿Tiene doble ciudadanía? \\
\hline 5. & ¿Pertenece a alguna asociación/organización civil de migrantes ecuatorianos? \\
\hline 7. & ¿Cuáles son sus principales preocupaciones sobre Ecuador en la actualidad? \\
\hline 8. & ¿Cree usted que los ecuatorianos residentes en el exterior deben tener repre- \\
\hline 9. & ¿Vontantes en la Asamblea Nacional de Ecuador? \\
\hline 10. & ¿Cómo calificaría la disposición de reservar asientos/curules en la Asamblea \\
& $\quad$ Nacional de Ecuador para la representación de sus ciudadanos residentes en \\
\hline 11. & ¿Cuál es su edad? \\
\hline 12. & ¿Cuál es su género? \\
\hline
\end{tabular}

Recibido para publicación en 18.04.2017 Aceptado para publicación en 28.06.2017 Received for publication in April 18 ${ }^{\text {th }}, 2017$ Accepted for publication in June 28 $8^{\text {th }}, 2017$ 